\title{
Kernel-Based Grouping of Histogram Data
}

\author{
Tilman Lange and Joachim M. Buhmann \\ Institue of Computational Science \\ ETH Zurich \\ 8092 Zurich, Switzerland \\ \{langet, jbuhmann\}@inf .ethz.ch
}

\begin{abstract}
Organizing objects into groups based on their co-occurrence with a second, relevance variable has been widely studied with the Information Bottleneck (IB) as one of the most prominent representatives. We present a kernel-based approach to pairwise clustering of discrete histograms using the Jensen-Shannon (JS) divergence, which can be seen as a two-sample test. This yields a cost criterion with a solid informationtheoretic justification, which can be approximated in polynomial time with arbitrary precision. In addition to that, a relation to optimal hard clustering IB solutions can be established. To our knowledge, we are the first to devise algorithms for the IB with provable approximation guaranties. In practice, one obtains convincing results in the context of image segmentation using fast optimization heuristics.
\end{abstract}

\section{Introduction}

Data clustering plays a central role in exploratory data analysis. Formally, one aims at finding a $k$-partition $C:[n] \rightarrow[k]$ 1] of a finite set $\mathcal{X}=\left\{x_{1}, \ldots, x_{n}\right\}$. Often, objects $X$ are characterized by their joint occurrence with an additional variable $Y, Y \in \mathcal{Y}=\left\{y_{1}, \ldots, y_{b}\right\}$ : E.g., text documents can be characterized by the words they contain. Image sites may be characterized by colors. In document categorization or image segmentation, this data can be summarized in a table of counts - each entry containing the number of joint occurrences of object (e.g. pixel or document) $x$ and variable (e.g. word or gray value) $y$. By normalization, one obtains the Maximum Likelihood (ML) estimate of the joint distribution $p(x, y)$. In clustering, one tries to organize the objects into classes based on the co-occurrence patterns. To this end, the normalized histograms $\hat{p}(y \mid x)$ are usually considered to characterize the objects $x \in \mathcal{X}$. Hence, clustering aims at determining the group structure such that empirically estimated distributions, that might have arisen from the same source, are grouped together. In this work, we introduce the Jensen-Shannon (JS) divergence as a kernel (sec. 2) and use it as distance for pairwise clustering of histograms (sec. 3). This clustering model is motivated by the observation that the JS divergence is a test for the two-sample problem [5]. As it is bounded, it has less pathologies in a pairwise setting than the classical Kullback-Leibler (KL) or the Jeffrey divergence.

${ }^{1}$ We use $[n]$ as a shorthand for the set $\{i \mid 1 \leq i \leq n\}$ throughout this work. 
A principled approach to address the problem of grouping histogram data represents the Information-Bottleneck (IB) introduced in [15] with the Histogram Clustering Model (HCM) 12 as a special case. The idea of the IB approach is to find a compact representation of the variable $X$ while preserving as much information as possible about a second (relevance) variable $Y$ - given the joint distribution $p(x, y)$. The mutual information, $I$, is here the performance measure of interest: the IB problem consists of finding cluster assignment probabilities $p(\nu \mid x)$ such that the functional $\mathcal{L}=\frac{1}{\beta} I(X, C)-I(Y, C)$ is minimized, where $\beta \geq 0$ is a Lagrange parameter. As the IB is intimately related to the JS divergence, we can develop a relationship to the IB for $\beta \rightarrow \infty$. Following [13], we use Euclidean embeddings to restate the pairwise as a $k$-means problem. Thereby, we can devise polynomial time approximation algorithms for pairwise clustering with JS divergences. We establish a relationship to the IB for the HCM case allowing us to extend the approximation results to the IB in the $\beta \rightarrow \infty$ limit - which is known to be $\mathcal{N} \mathcal{P}$-hard. These are the first rigorous approximation results for such clustering models. We also discuss optimization heuristics allowing quick operation in practice. In the experimental section, the applicability to image segmentation problems is demonstrated. We also show the possibility of simple data fusion in combination with kernel PCA-based de-noising leading to competitive solutions in comparison with $\mathrm{HCM}$ (sec. 4).

\section{The Jensen-Shannon-Kernel}

Let $\mathbf{p}=(p(y)) \in \mathbb{M}(\mathcal{Y})$ and $\mathbf{q}=(q(y)) \in \mathbb{M}(\mathcal{Y})$ be distributions over a discrete domain $\mathcal{Y}$ with $\mathbb{M}(\mathcal{Y})$ the set of distributions on $\mathcal{Y}$. The JS divergence is a symmetrized version of the KL divergence $d_{\mathrm{KL}}$ :

$$
\mathbb{R}_{\geq 0} \ni d_{\mathrm{JS}}(\mathbf{p} \| \mathbf{q}):=\frac{1}{2} d_{\mathrm{KL}}(\mathbf{p} \| \mathbf{m})+\frac{1}{2} d_{\mathrm{KL}}(\mathbf{q} \| \mathbf{m})
$$

with $\mathbf{m}=\frac{1}{2} \mathbf{p}+\frac{1}{2} \mathbf{q}$. Clearly, $d_{\mathrm{JS}}$ is symmetric, $>0$ iff $\mathbf{p} \neq \mathbf{q}$, and the square of a metric [6]. In fact, $d_{\text {JS }}$ represents a conditionally negative semi-definite kernel function [8]. An outline of the proof and a discussion of the issue of embeddings into vector spaces follows. Let $k$ be a conditionally positive definite (cpd) kernel. Then, $k$ can be transformed into a positive definite kernel function and there is a Hilbert space $\mathcal{H}$, such that $-k$ corresponds to squared 2-norm distances $\mathcal{H}$ [14. Hence, we show that $k=-d_{\mathrm{JS}}$ is cpd.

Definition 1 (e.g. in [14]). A symmetric function $k$ on $\mathbb{M}(\mathcal{Y}) \times \mathbb{M}(\mathcal{Y})$ is called conditionally positive definite (cpd) kernel if it is a positive semi-definite kernel for all $m \in \mathbb{N}$ and all $c_{i} \in \mathbb{R}$ with

$$
\sum_{i \in[m]} c_{i}=0 .
$$


Thus, we show that $d_{\mathrm{JS}}$ obeys $\sum_{i, j} c_{i} c_{j} d_{\mathrm{JS}}\left(\mathbf{p}_{i} \| \mathbf{p}_{j}\right) \leq 0$. for all $c_{i}, c_{j}$ fulfilling eq. 2 and for all $\mathbf{p}_{i}, \mathbf{p}_{j} \in \mathbb{M}(\mathcal{Y})$ (c.f. [1]). Now, note that

$$
d_{\mathrm{JS}}(\mathbf{p} \| \mathbf{q})=\frac{1}{2} \sum_{y \in \mathcal{Y}}\left[p(y) \log \left(\frac{2 p(y)}{p(y)+q(y)}\right)+q(y) \log \left(\frac{2 q(y)}{p(y)+q(y)}\right)\right]
$$

and, hence, if each summand is negative definite, then $d_{\mathrm{JS}}$ is. By expansion

$$
\begin{aligned}
\underbrace{\sum_{i, j} c_{i} c_{j} p_{i}(y) \log \left(2 p_{i}(y)\right)}_{=0} & +\underbrace{\sum_{i, j} c_{i} c_{j} q_{j}(y) \log \left(2 q_{j}(y)\right)}_{=0} \\
& -\sum_{i, j} c_{i} c_{j}\left(p_{i}(y)+q_{j}(y)\right) \log \left(p_{i}(y)+q_{j}(y)\right),
\end{aligned}
$$

since $\sum_{i} c_{i}=0$. Because $-z \log z$ is negative definite for $z \in \mathbb{R}_{+},-d_{\text {JS }}$ is cpd (see e.g. 1, p. 89 \& pp. 218-19). Next, we discuss how an embedding can be found using the empirical kernel map.

Let $\mathbf{I}_{n}$ be the $n \times n$ identity matrix and $\mathbf{e}_{n}=(1, \ldots, 1)^{\top}$. Let $\mathbf{Q}_{n}:=\mathbf{I}_{n}-\frac{1}{n} \mathbf{e}_{n} \mathbf{e}_{n}^{\top}$ be the centering matrix. The centered matrix $\tilde{\mathbf{K}}=-\frac{1}{2} \mathbf{Q}_{n} \mathbf{D}_{\text {JS }} \mathbf{Q}_{n}$, with $\mathbf{D}_{\text {JS }}=$ $\left(d_{\mathrm{JS}}\left(\mathbf{p}_{i} \| \mathbf{p}_{j}\right)\right)_{1 \leq i, j \leq n}$, is positive definite (prop. 8 in [14]), since $\mathbf{K}=-\mathbf{D}_{\text {JS }}$ is cpd, and, hence, $\mathbf{D}_{\text {JS }}$ must derive from squared Euclidean distances (thm. 1 in [13]). Kernel Principal Component Analysis (kPCA) can be used to find a $(n-1)$ dimensional, isometric embedding in $\left(\mathbb{R}^{n-1},\|\cdot\|\right)$ (cf. [13]): Let $\tilde{\mathbf{K}}=\mathbf{U} \boldsymbol{\Lambda} \mathbf{U}^{\top}$ be an eigen-decomposition of $\tilde{\mathbf{K}}$ with $\mathbf{U}=\left(u_{1}, \ldots, u_{n}\right)$ and $\boldsymbol{\Lambda}=\operatorname{diag}\left(\lambda_{1}, \ldots, \lambda_{n}\right)$, $\lambda_{i} \geq \lambda_{j}$, for $i \leq j$. Then, a $d$-dimensional embedding, $d \leq n-1$, can be found by $\mathbf{X}:=\mathbf{U}_{d}\left(\boldsymbol{\Lambda}_{d}\right)^{1 / 2}$ with $\mathbf{U}_{d}=\left(u_{1}, \ldots, u_{d}\right)$ and $\boldsymbol{\Lambda}_{d}=\operatorname{diag}\left(\lambda_{1}, \ldots, \lambda_{d}\right)$. Taking $d<n-1$ comes at the expense of distorting the inter-point distances in the embedding space. However, de-noising of the original data can be achieved this way which can be advantageous for clustering 13 . For $d=n-1$, using kPCA an $(n-1)$-dimensional, isometric embedding into real vector space can be found such that squared Euclidean distances in the embedding space correspond to JS divergences in $\mathbb{M}(\mathcal{Y})$.

\section{Pairwise Clustering with the JS-Kernel}

The problem of Pairwise Clustering [9] can be stated as follows: Given an $n \times n$ matrix $\mathbf{D}=\left(D_{i j}\right)$ of pairwise dissimilarities between objects $i$ and $j$, find an assignment $C:[n] \rightarrow[k]$ of objects to classes such that

$$
h^{\mathrm{pw}}(\mathbf{D} ; C)=\frac{1}{2} \sum_{\nu \in[k]} \frac{1}{n_{\nu}} \sum_{i, j \in C^{-1}(\nu)} D_{i j}
$$

is minimized where $n_{\nu}:=\left|C^{-1}(\nu)\right|$ with $C^{-1}(\nu)=\{i \in[n] \mid C(i)=\nu\} . h^{\text {pw }}$ measures the avg. within-cluster dissimilarity and aims at finding compact clusters.

In histogram grouping, a problem instance consists of $n$ empirical distributions $\mathbf{p}_{x}=(\hat{p}(y \mid x))_{y \in \mathcal{Y}}$ assuming $p(x)=\frac{1}{n}, x \in \mathcal{X}$. Here, $d_{\text {JS }}$ represents a natural 
measure for testing if two empirical distributions $\mathbf{p}$ and $\mathbf{q}$ might have arisen from the same source: the authors of [5] quantified the probability that the (sequence of) measurements for objects $x_{i}$ and $x_{j}$ were drawn from the same distribution by the method of types [3]. Using the JS divergence as dissimilarity $D_{i j}=d_{\mathrm{JS}}\left(\mathbf{p}_{x_{i}} \| \mathbf{p}_{x_{j}}\right)$ for pairwise histogram grouping is, hence, natural, as objects are grouped together that are likely to have the same origin.

The pairwise clustering criterion $h^{\mathrm{pw}}$ can be rephrased as a $k$-means problem (cf. 13]) if pairwise dissimilarities correspond to squared Euclidean distances. We exploit this to get approximation guaranties for pairwise grouping with JS divergences. The results are extended to the first approximation guaranties for the IB in the literature.

Approximation Results: JS divergences correspond to squared distances in a real Hilbert space (cf. sec. 2). Thus, there is a function $\phi$ mapping distributions $\mathbf{p}$ to $\phi(\mathbf{p})$ in that space. Hence, one obtains for $h^{\mathrm{km}}$ and $h^{\mathrm{pw}}$ (cf. [13]):

$$
h^{\mathrm{pw}-\mathrm{js}}=\sum_{\nu, i, j} \frac{1}{2 n_{\nu}} \overbrace{\left\|\phi\left(\mathbf{p}_{x_{i}}\right)-\phi\left(\mathbf{p}_{x_{j}}\right)\right\|^{2}}^{=d_{\mathrm{JS}}\left(\mathbf{p}_{x_{i}} \| \mathbf{p}_{x_{j}}\right)}=h^{\mathrm{km}}=\sum_{i \in[n]}\left\|\phi\left(\mathbf{p}_{x_{i}}\right)-\mathbf{z}_{C(i)}\right\|^{2},
$$

where $\mathbf{z}_{\nu}=\frac{1}{n_{\nu}} \sum_{i \in C^{-1}(\nu)} \phi\left(\mathbf{p}_{x_{i}}\right)$ is the centroid in feature space. An isometric embedding into a real vector space based on the empirical kernel map can be computed by the procedure discussed in sec. 2 (cf. [13). Optimizing $h^{\mathrm{pw}}$ with pairwise JS divergences and minimizing $h^{\mathrm{km}}$ on the embedded data are, thus, equivalent problems. Hence, every approximation algorithm for $h^{\mathrm{km}}$ is one for $h^{\mathrm{pw}-\mathrm{js}}$. There is, e.g., a polynomial time approximation scheme (PTAS) for the $k$ means objective function [11. Hence, one can get $\epsilon$-close, $\forall \epsilon>1$, to the optimal solution of $h^{\mathrm{km}}$ in running time polynomial in $n$. A 2-approximation can also be obtained by a $k$-dimensional embedding (via $\mathrm{kPCA}$ ) of the data: an optimal solution in the $k$-dimensional subspace is known to yield a 2 -approximation for the $(n-1)$-dimensional problem 4 . Finding the optimal solution by enumerating all Voronoi partitions in $\mathbb{R}^{k}$ takes $O\left(n^{k^{2}+1}\right)$ steps (cf. [10]), and, hence, one obtains a polynomial time 2-approximation algorithm.

Theorem 2. If $\mathbf{D}_{J S}$ is a matrix of pairwise JS divergences, there is a PTAS for $h^{p w}\left(\mathbf{D}_{J S} ; C\right)$. Using $k P C A$, there is also a 2-approximation for $h^{p w}\left(\mathbf{D}_{J S} ; C\right)$.

Relation to the Information Bottleneck: One motivation for studying the JS kernel for clustering stems from its close relationship to the Information Bottleneck (IB) in its hard clustering version, i.e. the Histogram Clustering Model (HCM) (cf. [12]). Here, we develop a connection between hard-clustering IB/HCM and Pairwise Clustering with the JS kernel yielding approximation guaranties for the hard-clustering IB case.

The HCM cost function is related to the IB as follows: Suppose, $C:[n] \rightarrow[k]$ is a hard clustering, then $I(X, C)=H(C)$ holds, since $p(\nu \mid x) \in\{0,1\}$, for $\nu \in[k]$. 
Now consider $I(Y, C)$ : With $\mathbf{q}_{\nu}=(p(y \mid \nu))_{y \in \mathcal{Y}}$, where $p(y \mid \nu)=\sum_{x} p(y \mid x) p(x \mid \nu)$ one obtains for the second term of the IB functional

$$
I(Y, C)=-\sum_{\nu, i} p\left(x_{i}, \nu\right) d_{\mathrm{KL}}\left(\mathbf{p}_{x_{i}} \| \mathbf{q}_{\nu}\right)+\text { const. }
$$

with the second term being a data-dependent constant. Assuming that $p(x)=$ $1 / n$ and $p(\nu \mid x) \in\{0,1\}$, one gets in the $\beta \rightarrow \infty$-limit after dropping the constant the HCM cost function:

$$
h^{\mathrm{hcm}}\left(\left\{\mathbf{p}_{x_{i}} \mid i \in[n]\right\} ; C\right):=\frac{1}{n} \sum_{\nu \in[k]} \sum_{i \in C^{-1}(\nu)} d_{\mathrm{KL}}\left(\mathbf{p}_{x_{i}} \| \mathbf{q}_{\nu}\right) .
$$

A relationship between $h^{\mathrm{hcm}}$ and $h^{\mathrm{pw}-\mathrm{js}}$ is now established. First note, that we have $d_{\mathrm{JS}} \leq \frac{1}{2} d_{\mathrm{KL}}$ (eq. (9) in [16]). Consider now

$$
h^{\mathrm{cjs}}\left(\left\{\mathbf{p}_{x_{i}} \mid i \in[n]\right\} ; C\right)=\frac{1}{n} \sum_{\nu \in[k]} \sum_{i \in C^{-1}(\nu)} d_{\mathrm{JS}}\left(\mathbf{p}_{x_{i}} \| \mathbf{q}_{\nu}\right)
$$

obtained by replacing $d_{\mathrm{KL}}$ with $d_{\mathrm{JS}}$ in $h^{\mathrm{hcm}}$. Clearly, $h^{\mathrm{cjs}} \leq \frac{1}{2} h^{\mathrm{hcm}}$ and $n h^{\mathrm{cjs}} \leq$ $2 h^{\mathrm{pw}-\mathrm{js}}$ holds, by the joint convexity of the JS divergence [2. Also from [16]:

$$
2 h^{\mathrm{pw}-\mathrm{js}}-n h^{\mathrm{cjs}}=\frac{n}{2} h^{\mathrm{hcm}}-\alpha \geq 0 \Leftrightarrow 2 h^{\mathrm{pw}-\mathrm{js}}-\frac{n}{2} h^{\mathrm{hcm}}+\alpha=n h^{\mathrm{cjs}} \geq 0
$$

for some $\alpha \geq 0$. Hence, we finally arrive at a lower bound on $h^{\text {hcm }}$ in terms of $h^{\mathrm{pw}-\mathrm{js}}$ :

$$
h^{\mathrm{cjs}} \leq \frac{1}{2} h^{\mathrm{hcm}} \Rightarrow h^{\mathrm{pw}-\mathrm{js}} \leq \frac{n}{2} h^{\mathrm{hcm}} .
$$

We now look for an upper bound: At first, we note that for any two distributions $\mathbf{p}$ and $\mathbf{q}$, we have

$$
d_{\mathrm{KL}}(\mathbf{p} \| \mathbf{q}) \leq 2 d_{\mathrm{JS}}(\mathbf{p} \| \mathbf{q})+\log \left(\frac{1}{2}(1+\gamma)\right),
$$

where $\gamma=\max _{y} \frac{p(y)}{q(y)}$ (thm. 4 in [16]). For any solution of $h^{\text {hcm }}$, assuming $p(y \mid \nu)=$ $1 / n_{\nu} \sum_{i \in C^{-1}(\nu)} \hat{p}\left(y \mid x_{i}\right): \gamma$ is bounded for $d_{\mathrm{KL}}\left(\mathbf{p}_{x_{i}} \| \mathbf{q}_{\nu}\right)$ if only quotients with $p(y \mid \nu) \neq 0$ are considered. We can neglect the other case because if $p(y \mid \nu)=0$, then $\hat{p}\left(y \mid x_{i}\right)=0$ and, thus, $0 \log \frac{0}{0}=0$. Let $\bar{\gamma}:=\max _{\nu} \max _{i \in C^{-1}(\nu)} \log \left(\frac{1}{2}(1+\right.$ $\left.\left.\max _{y}\left\{\frac{\hat{p}\left(y \mid x_{i}\right)}{p(y \mid \nu)} \mid p(y \mid \nu) \neq 0\right\}\right)\right)$. Clearly, $\bar{\gamma}$ is also finite. Then,

$$
\frac{2}{n} h^{\mathrm{pw}-\mathrm{js}} \leq h^{\mathrm{hcm}} \leq 2 h^{\mathrm{cjs}}+\bar{\gamma} \leq 4 \frac{h^{\mathrm{pw}-\mathrm{js}}}{n}+\bar{\gamma} .
$$

Intuitively, it is clear that an optimal solution for $h^{\mathrm{hcm}}$ minimizes $\bar{\gamma}$, since a large $\bar{\gamma}$ corresponds to a large cost contribution. Thereby, we arrive at the following

Theorem 3. Let $\left\{\mathbf{p}_{x_{i}} \mid i \in[n]\right\}$ be a fixed instance and let $C^{*}$ be an optimal solution for $h^{h c m}$. Then, if $C$ is an $\epsilon$-approximate solution for $h^{p w-j s}$, the costs w.r.t. $h^{h c m}$ can be bounded from above by $2 \varepsilon h^{h c m}\left(C^{*}\right)+\bar{\gamma} \geq h^{h c m}(C)$. 
Thus, by efficiently approximating $h^{\mathrm{pw}-\mathrm{js}}$, we find a good approximate solution to the hard clustering IB / HCM cost function. To the knowledge of the authors, this is the first approximation guaranty for HCM in the literature.

Heuristic Optimization: Although it is pleasing to derive an algorithm that produces results of guaranteed quality, such algorithms still might be impractical since they may require unacceptable computation time, especially for large $k$. We, thus, resort to the classical $k$-means algorithm applied to the data embedded into $\mathbb{R}^{k}$ using kPCA. The embedding represents a major computational burden, as it requires the computation of an eigenbasis usually taking $O\left(n^{3}\right)$ steps. In practice, this can be significantly sped up by first selecting $m \ll n$ randomly sampled objects to compute an approximate eigenbasis and then use this basis to get a representation of the full data set in the embedding space. This is achieved using the technique in [13] which is the Nyström extension that has been successfully applied in the spectral clustering context (see [7]).2 The running time for the embedding step can be reduced from $O\left(n^{3}\right)$ to $O\left(m^{2} n\right)$ being linear in $n$ if $m=$ const. In total, one gets a running time of $O\left(m^{2} n+s n k^{2}\right)(s=\#$ iterations needed by $k$-means). This is linear in the number of objects in each iteration for constant $k$ and $m$.

\section{Experimental Results}

We provide experimental evidence for the usefulness of $h^{\mathrm{pw}-\mathrm{js}}$ and compare our results with those obtained with HCM. For the optimization of $h^{\mathrm{pw}-\mathrm{js}}$, we used $k$-means on the data embedded in a $k$-dimensional subspace. The required eigenbasis has been computed on a random sub-sample of size $m=500$ (less than $10 \%$ of the full data set) and the Nyström extension technique was employed to embed the whole data. For HCM, a multi-scale annealing implementation was used. The number of classes was set to $k=5$ for the toy image and to $k=3$ for the real world image. We extracted 12-bin gray-value histograms from the image data on a grid of $128 \times 128$ image sites. Gabor feature histograms (with 12 bins/channel) for 4 orientations and 3 different scales of the Gabor wavelet were computed. The Gabor feature histograms were then stacked into a single histogram consisting of 144 bins. By suitable re-normalization one obtains empirical probability distributions.

A Toy Example: On the left side of fig. 1(a), an artificial image is shown with 5 segments: 3 segments are characterized by their texture while 2 of the segments are characterized by intensity. We added Gaussian noise to the image to make the segmentation task more difficult. All 5 classes cannot be separated based on either only gray-value or texture information. The information contained in

\footnotetext{
$\overline{{ }^{2}}$ Let $\mathbf{D}_{\text {JS }}$ be the matrix for which the embedding was computed and $\mathbf{D}_{\text {JS }}^{\text {new }}$ be the $n-m \times m$ matrix with JS divergences between new and already known objects. Set $\tilde{\mathbf{K}}^{\text {new }}:=-\frac{1}{2}\left(\mathbf{D}_{\mathrm{JS}}^{\text {new }} \mathbf{Q}_{m}-\frac{1}{m} \mathbf{e}_{n-m} \mathbf{e}_{m}^{\top} \mathbf{D}_{\mathrm{JS}} \mathbf{Q}_{m}\right)$. Then the embedding in $d$ dimensions can be obtained by $\mathbf{X}^{\text {new }}=\tilde{\mathbf{K}}^{\text {new }} \mathbf{U}_{d}\left(\boldsymbol{\Lambda}_{d}\right)^{-1 / 2}$.
} 
the gray-value and in the Gabor histograms was, thus, combined by averaging the JS divergences obtained from both information sources. As the sum of two kernels is a kernel, the algorithms mentioned above can still be applied to perform segmentation (a trace normalization was also applied to the matrices). On the right of figure $1(\mathrm{a})$ the result for $k=5$ on this data set is shown, which identifies all segments correctly. A similar combination can be obtained with HCM as well but proves itself to be less efficient. The HCM segmentation, given in the middle of fig. 1(a), was obtained by treating all channels independently, i.e. the costs $\frac{1}{l n} \sum_{j \in[l]} \sum_{\nu \in[k]} \sum_{i \in C^{-1}(\nu)} d_{\mathrm{KL}}\left(\mathbf{p}_{x_{i}}^{(j)} \| \mathbf{q}_{\nu}^{(j)}\right)$ were considered, where $j$ runs over the $l=12+1$ different (one intensity and 12 texture) channels. This strategy obviously produces poorer results 3
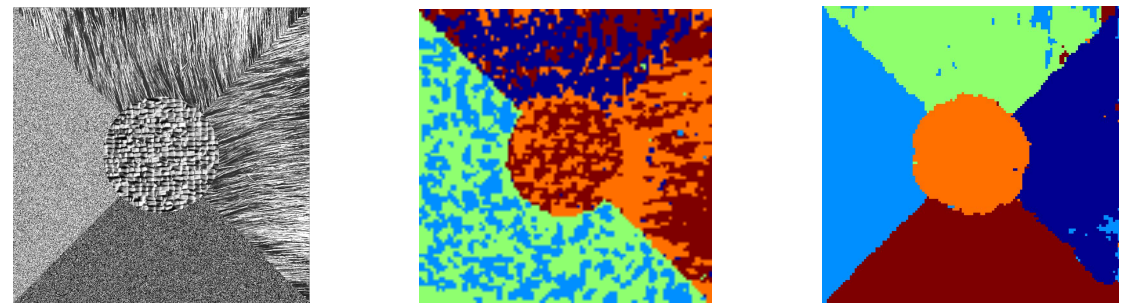

(a) Toy Image Data: (left) Original Image, (middle) segmentation by HCM, (right) JS Pairwise Clustering
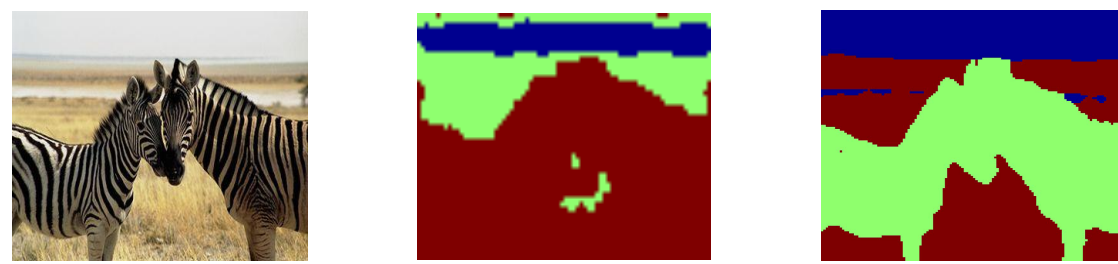

(b) A real world example: Toy Image Data: (left) Original Image, (middle) Segmentation by HCM, (right) JS Kernel Clustering

Fig. 1. Experimental results on the image data

Segmentation of Real World Images: On the left of figure 1(b) a real word image depicting two zebras is shown. Again, HCM and Pairwise Clustering with $d_{\mathrm{JS}}$ were used. Obviously, segmentations obtained with JS divergences on the combined data are smoother than those of HCM. In contrast to the HCM result, the segmentation based on $h^{\mathrm{pw}-\mathrm{js}}$ nicely separates the zebras from the two background classes. The use of $\mathrm{kPCA}$ for de-noising turned out to be an important component as it emphasizes the group structure in this example.

${ }^{3}$ In principle one could resort to a distribution for the joint occurrence of a gray value and a certain Gabor response. However, this implies a severe estimation problem. 


\section{Conclusions}

This work discussed the grouping of histogram data using pairwise clustering with JS divergences. Theoretical results demonstrate that the JS kernel renders the pairwise clustering problem tractable: efficient approximation algorithms can be devised and fast heuristics exist. A connection to HCM as a special case of IB was established. The experimental section demonstrated that our approach can be useful in image segmentation: Segmentations obtained with $h^{\mathrm{pw}-\mathrm{js}}$ were more meaningful than those generated using $h^{\mathrm{hcm}}$. Particularly noteworthy is the possibility to fuse data in a straightforward way allowing for better segmentation results in practice.

\section{References}

1. Berg, C., Christensen, J.P.R., Ressel, P.: Harmonic Analysis on Semigroups. Springer, Heidelberg (1984)

2. Burbea, J., Rao, C.R.: On the convexity of some divergence measures based on entropy functions. IEEE T-IT 28(3) (1982)

3. Cover, T.M., Thomas, J.A.: Elements of Information Theory. Wiley Series in Telecommunications. John Wiley \& Sons, New York (1991)

4. Drineas, P., Frize, A., Kannan, R., Vempala, S., Vinay, V.: Clustering in large graphs and matrices. Technical report, Yale University (1999)

5. El-Yaniv, R., Fine, S., Tishby, N.: Agnostic classification of markovian sequences. In: NIPS 10 (1998)

6. Endres, D., Schindelin, J.: A new metric for probability distributions. IEEE TIT 49, 1858-1860 (2003)

7. Fowlkes, C., Belongie, S., Chung, F., Malik, J.: Spectral grouping using the nyström method. IEEE T-PAMI 26, 214-225 (2004)

8. Fuglede, B., Topsœ, F.: Jensen-shannon divergence and hilbert space embedding. In: Proc. ISIT (2004)

9. Hofmann, T., Buhmann, J.M.: Pairwise data clustering by deterministic annealing. IEEE T-PAMI 19 (1997)

10. Inaba, M., Katoh, N., Ima, H.: Applications of weighted voronoi diagrams and randomization to variance-based $k$-clustering. In: 10th Computational Geometry (1994)

11. Ostrovsky, R., Rabani, Y.: Polynomial time approximation schemes for geometric min-sum median clustering. JACM 49, 139-156 (2002)

12. Pereira, F., Tishby, N., Lee, L.: Distributional clustering of English words. In: 31st ACL (1993)

13. Roth, V., Laub, J., Kawanabe, M., Buhmann, J.M.: Optimal cluster preserving embedding of nonmetric proximity data. IEEE T-PAMI 25, 1540-1551 (2003)

14. Schölkopf, B.: The kernel trick for distances. In: NIPS 12 (2000)

15. Tishby, N., Pereira, F.C., Bialek, W.: The information bottleneck method. In: 37th ACL (1999)

16. Topsøe, F.: Some inequalities for information divergence and related measures of discrimination. IEEE T-IT 46, 1602-1609 (2000) 